\title{
Integrated Bioinformatics Analysis of mRNAs and miRNAs Identified Potential Biomarkers of Oral Squamous Cell Carcinoma
}

\author{
Nasrin Amiri-Dashatan', Mehdi Koushki², Ali Jalilian², Nayeb Ali Ahmadi², \\ Mostafa Rezaei-Tavirani ${ }^{3 *}$
}

\begin{abstract}
Background: Oral cancer is a frequently encountered neoplasm of the head and neck region, being the eighth most common type of human malignancy worldwide. Despite improvement in its control, morbidity and mortality, rates have improved little in the past decades. The present investigations about gene interaction and pathways still could not clear the appearance and development of oral squamous cell carcinoma (OSCC), completely. The aim of this study is to investigate the key genes and microRNAs interaction in OSCC. Materials and Methods: The microarray datasets GSE13601 and GSE98463, including mRNA and miRNA profiles, were extracted from the GEO database and were analyzed using GEO2R. Functional and pathway enrichment analyses were performed by using the DAVID database. The protein-protein interaction (PPI) network was constructed and analyzed using STRING database and Cytoscape software, respectively. Finally, miRDB was applied to predict the targets of the differentially expressed miRNAs (DEMs). Results: Totally, 97 differentially expressed genes (DEGs) were found in OSCC, including 66 up-regulated and 31 down-regulated genes. The gene ontology (GO) and Kyoto Encyclopedia of Genes and Genomes (KEGG) pathway enrichment analyses showed that up-regulated genes were significantly enriched in movement of cell or subcellular component, cell adhesion, biological adhesion, cellular localization, apoptotic signaling pathway, while the downregulated genes were enriched in muscle system process and oxidation-reduction process. From the PPI network, the top 10 nodes with the highest degree were detected as hub genes. In addition, 18 DEMs were screened, which included 7 up-regulated and 11 down-regulated miRNAs. STAT 1 was potentially targeted by three miRNAs, including has-miR6825-5P, has-miR-4495, and has-miR-5580-3P. Conclusion: The roles of DEMs such as hsa-mir-5580-3p in OSCC through interactions with DEGs CD44, ACLY, ACTR3, STAT1, LAMC2 and YWHAZ may offer a suitable candidate biomarker pattern for diagnosis, prognosis and treatment processes in OSCC.
\end{abstract}

Keywords: Oral squamous cell carcinoma- microarray analysis- DEGs- miRNA- protein-protein interaction network

Asian Pac J Cancer Prev, 21 (6), 1841-1848

\section{Introduction}

Oral squamous cell carcinoma (OSCC) is the sixth most common cancer type worldwide. Despite advances in treatment and surgical methods, the 5-year survival rate for patients with oral cancer did not show improvement in the last two decades (Malik et al., 2016). The incidence of OSCC is also increasing in recent years, especially in younger people. Therefore, further studies are needed to better understanding of molecular mechanisms underlying this disease. Recently, several studies have reported that changes of gene expression levels is related to formation and progression of OSCC tumors, however, there is an urgent need to identify potential mRNA biomarkers for prediction, early diagnosis and treatment of oral tumors (Chakraborty et al., 2010; Ge et al., 2015). MicroRNAs (miRNAs) are small, noncoding RNAs with 18-25 nucleotides long that regulate protein expression at the post-transcriptional level. MiRNAs involvement in various physiological processes including cell proliferation, differentiation, apoptosis, and angiogenesis has been reported (Malik et al., 2016; Park et al., 2017). The abnormal expression of specific miRNAs can lead to cancer progression (Momen-Heravi and Bala, 2018). The use of microRNAs as diagnostic and prognostic markers in diseases has been considered in recent years. Several studies have reported miRNAs profiling and differentially expressed miRNAs in OSCC compared to normal tissue (Chen et al., 2008; Manikandan et al., 2016; Schneider et al., 2018). Today, bioinformatics and computational 
methods play an important role in biological studies, especially in protein and gene regulatory network analysis that led to identify key genes and proteins in different diseases pathogenesis (Atan et al., 2014; Atan et al., 2018; Dashatan et al., 2018). This study aimed to assess the mRNA-miRNA network and pathway enrichment analysis on extracted genes and miRNAs from GEO database to find potential molecular mechanisms associated with oral cancer.

\section{Materials and Methods}

\section{Microarray data collection}

We searched the GEO database (https://www. ncbi.nlm. nih.gov/geo/) using the following keywords: "Oral Squamous cell carcinoma" (study keyword), "Homo sapiens" (organism), "Expression profiling by array" (study type), and "tissue" (attribute name). After a systematic review, two gene expression profiles (GSE13601 and GSE98463) were collected for analysis. Above all, the differentially expressed mRNAs between patients with oral squamous cell carcinoma (OSCC) tissue $(n=31)$ and in those with normal tongue tissue $(n=26)$ were identified by GEO2R from the GSE13601 microarray. The differentially expressed miRNAs between oral squamous cell carcinoma (OSCC) $(n=8)$ and oral mucosa $(n=8)$ were identified by GEO2R from the GSE98463 microarray dataset (Table 1).

\section{Differential expression of miRNAs and $m R N A s$}

After GEO2R was applied to analysis differentially expressed mRNAs and miRNAs between OSCC and normal tissue, the differentially expressed mRNAs were selected with a criterion of adj. $\mathrm{p}<0.05$ and $\log$ fold change (FC) $\geq 2$.

Functional annotation and pathway enrichment analysis

Gene ontology (Go) including biological pathway, molecular function, and cellular component and KEGG pathway analyses of the DEGs were performed using the DAVID database.

\section{Protein-protein interaction network analysis}

DEGs $(\mathrm{FC}>2$ and $\mathrm{P}<0.05)$ were used for protein interaction network instruction. Protein- protein interaction network was constructed by using STRING database (http://string-db.org). The protein-protein interaction network was visualized and analyzed using the Cytoscape software version 3.6.0. Cytoscape is an open source software that integrates biomolecular interaction networks with large-scale expression data into a unified conceptual framework. The top 10 high node degree genes was screened as hub genes. Clustering analysis of protein network was performed in order to detect modules by using MCODE (Molecular Complex Detection) algorithm with haircut on, node score cut-off $=0.2, \mathrm{k}$-core $=2$, and $\max$. Depth $=100$.

\section{Prediction of miRNAs targets}

The target genes of the DEMs from GSE98463 were predicted with TargetScan Human database (www. targetscan.org), which is an online database for predicting microRNA targets.

\section{Results}

\section{Identification of DEGs}

A total of 97 (including 66 up-regulated and 31 down-regulated) genes and 18 (including 7 up-regulated and 11 down-regulated) miRNAs with a threshold p-value $<0.05$ and $\mathrm{FC} \geq 2$ were considered as significantly differentially expressed (Supplementary Table 1). The full list of the genes contained in these datasets is shown in Table 1. The most significantly down-regulated mRNA was MYOC (Supplementary Table 1). Significant up-regulation of MMP1 was detected in OSCC tissue samples compared with normal tongue tissue. According to supplementary Table 2, has-miR-1290 and has-miR5580-3p were detected as significantly up-regulated and significantly down-regulated miRNAs between OSCC samples compared with normal healthy sample.

\section{Functional and pathway enrichment analyses}

According to Table 2, the GO term enrichment analysis showed that in biological processes-associated category, the up-regulated genes were significantly enriched in movement of cell or subcellular component, cell adhesion, biological adhesion, etc, while the down-regulated genes were enriched in muscle system process and oxidation-reduction process. In addition, the cellular component analysis showed that adherence junction and mitochondrial membrane were mainly enriched category in up-regulated and down-regulated genes, respectively. Moreover, for molecular function, the up-regulated genes were enriched in actin binding, and the down-regulated genes were enriched in cofactor binding and DNA binding. As shown in Table 2, the KEGG pathway analysis indicated that the micoRNAs in cancer, pathway in cancer, FC gamma R-mediate phagocytosis were mainly enriched pathways in up-regulated genes, while two pathways including phenylalanine metabolism and tyrosine metabolism were overrepresented in down-regulated genes.

\section{PPI network and modules analysis}

Sixty-five nodes and 108 edges were mapped in the PPI

Table 1. Characteristics of mRNA and miRNA Expression Profiling of Oral Squamous Cell Carcinoma (OSCC)

\begin{tabular}{lllll}
\hline GEO ID & Platform & $\begin{array}{l}\text { Samples } \\
\text { (cases/controls) }\end{array}$ & Country & References \\
\hline GSE13601 & $\begin{array}{l}\text { GPL8300 [HG_U95Av2] Affymetrix Human Genome } \\
\text { U95 Version 2 Array }\end{array}$ & $31 / 26$ & USA & Estilo CL et al. (Estilo et al., 2009) \\
GSE98463 & $\begin{array}{l}\text { GPL21572 [miRNA-4] Affymetrix Multispecies } \\
\text { miRNA-4 Array [ProbeSet ID version] }\end{array}$ & $8 / 8$ & Spain & $\begin{array}{l}\text { Chamorro-Petronacci C et al. } \\
\text { (Chamorro-Petronacci et al., 2018a) }\end{array}$ \\
\hline
\end{tabular}


Table 2. Functional and Pathway Enrichment Analysis of Up-Regulated and Down-Regulated Genes in Oral Squamous Cell Carcinoma (OSCC) Tissue

\begin{tabular}{|c|c|c|c|}
\hline Category & Term & Count & $P$-value \\
\hline \multicolumn{4}{|l|}{ Up-regulated } \\
\hline GOTERM_BP_FAT & GO:0006928 movement of cell or subcellular component & 20 & $5.70 \mathrm{E}-06$ \\
\hline GOTERM_BP_FAT & GO:0007155 cell adhesion & 19 & $1.10 \mathrm{E}-05$ \\
\hline GOTERM_BP_FAT & GO:0022610 biological adhesion & 19 & $1.10 \mathrm{E}-05$ \\
\hline GOTERM_BP_FAT & GO:0051641 cellular localization & 23 & $2.10 \mathrm{E}-05$ \\
\hline GOTERM_BP_FAT & GO:0097190 apoptotic signaling pathway & 11 & $2.90 \mathrm{E}-05$ \\
\hline GOTERM_CC_FAT & GO:0005912 adherens junction & 19 & $1.00 \mathrm{E}-10$ \\
\hline GOTERM_CC_FAT & GO:0070161 anchoring junction & 19 & $1.50 \mathrm{E}-10$ \\
\hline GOTERM_CC_FAT & GO:0070062 extracellular exosome & 30 & $1.50 \mathrm{E}-07$ \\
\hline GOTERM_CC_FAT & GO:1903561 extracellular vesicle & 30 & $1.60 \mathrm{E}-07$ \\
\hline GOTERM_CC_FAT & GO:0043230 extracellular organelle & 30 & $1.70 \mathrm{E}-07$ \\
\hline GOTERM_MF_FAT & GO:0003779 actin binding & 11 & $1.90 \mathrm{E}-06$ \\
\hline GOTERM_MF_FAT & GO:0008092 cytoskeletal protein binding 15 & 15 & $2.00 \mathrm{E}-06$ \\
\hline GOTERM_MF_FAT & GO:0051015 actin filament binding & 7 & $9.70 \mathrm{E}-06$ \\
\hline GOTERM_MF_FAT & GO:0050839 cell adhesion molecule binding & 10 & $5.00 \mathrm{E}-05$ \\
\hline GOTERM_MF_FAT & GO:0098641 cadherin binding involved in cell- cell adhesion & 7 & 7.30E-04 \\
\hline KEGG_PATHWAY & hsa05206:MicroRNAs in cancer & 6 & $2.40 \mathrm{E}-02$ \\
\hline KEGG_PATHWAY & hsa05200:Pathways in cancer & 7 & $2.50 \mathrm{E}-02$ \\
\hline KEGG_PATHWAY & hsa04666:FC gamma R-mediated phagocytosis & 3 & $8.50 \mathrm{E}-02$ \\
\hline KEGG_PATHWAY & hsa04512:ECM-receptor interaction & 3 & $9.10 \mathrm{E}-02$ \\
\hline \multicolumn{4}{|l|}{ Down-regulated } \\
\hline GOTERM_BP_FAT & GO:0003012 muscle system process & 6 & $3.60 \mathrm{E}-04$ \\
\hline GOTERM_BP_FAT & GO:0055114 oxidation-reduction process & 8 & $6.00 \mathrm{E}-04$ \\
\hline GOTERM_BP_FAT & GO:0010765 positive regulation of sodium ion transport & 3 & $1.40 \mathrm{E}-03$ \\
\hline GOTERM_BP_FAT & GO:0006732 coenzyme metabolic process & 5 & $1.40 \mathrm{E}-03$ \\
\hline GOTERM_BP_FAT & GO:0006082 organic acid metabolic process & 3 & $2.00 \mathrm{E}-03$ \\
\hline GOTERM_CC_FAT & GO:0031966 mitochondrial membrane & 8 & $1.60 \mathrm{E}-04$ \\
\hline GOTERM_CC_FAT & GO:0005743 mitochondrial inner membrane 7 & 7 & $2.00 \mathrm{E}-04$ \\
\hline GOTERM_CC_FAT & GO:0005740 mitochondrial envelope & 8 & $2.30 \mathrm{E}-04$ \\
\hline GOTERM_CC_FAT & GO:0019866 organelle inner membrane & 7 & $3.70 \mathrm{E}-04$ \\
\hline GOTERM_CC_FAT & GO:0005739 mitochondrion & 11 & $4.20 \mathrm{E}-04$ \\
\hline GOTERM_MF_FAT & GO:0048037 cofactor binding & 6 & $8.00 \mathrm{E}-05$ \\
\hline GOTERM_MF_FAT & GO:0051287 DNA binding & 4 & $9.30 \mathrm{E}-05$ \\
\hline GOTERM_MF_FAT & GO:0050662 coenzyme binding & 5 & $2.40 \mathrm{E}-04$ \\
\hline GOTERM_MF_FAT & GO:0004367 glycerol-3-phosphate dehydrogenase activity & 2 & $3.40 \mathrm{E}-03$ \\
\hline GOTERM_MF_FAT & GO:0016616 oxidoreductase activity & 3 & $1.60 \mathrm{E}-02$ \\
\hline KEGG_PATHWAY & hsa00360:Phenylalanine metabolism & 2 & $4.60 \mathrm{E}-02$ \\
\hline KEGG_PATHWAY & hsa00350:Tyrosine metabolism & 2 & $9.20 \mathrm{E}-02$ \\
\hline
\end{tabular}

network of identified DEGs, including 48 up-regulated genes and 17 down-regulated genes (Figure 1a). The top 10 nodes with the higher degrees screened as hub genes are ENO1, CD44, ACLY, ACTR3, STAT1, MYH11, $L A M C 2, Y W H A Z, Y W H A Q$, and HIF1A as shown in Table 3. A significant module including 16 nodes and 20 edges was obtained using MCODE which shown in Figure $1 \mathrm{~b}$. Biological process enrichment analysis results of module genes have shown in Table 4.
miRNAs targets results

In this study, top 10 DEMs were identified including 5 up-regulated miRNAs and 5 down-regulated miRNAs. The TargetScan Human database was used to predict target genes of the identified DEMs as shown in Table 5 . The has-miR-1290, one of the significantly up-regulated miRNAs, was found to target ACTR3, MYH11, and $Y W H A Z$. At the other hand, has-miR-5580-3p, the main down-regulated miRNAs, potentially targeted $C D 44$, ACLY, ACTR3, STAT1, LAMC2 and YWHAZ. Moreover, we found that ACLY, STAT1 and YWHAZ was potentially 
Table 3. Top 10 Hub Genes and Related DEMs

\begin{tabular}{lcl}
\hline Gene symbol & Node degree & \multicolumn{1}{c}{ Related DEMs } \\
\hline ENO1 & 12 & has-miR-6825-5P \\
CD44 & 9 & has-miR-7111-5P, has-miR-5580-3P \\
ACLY & 8 & has-miR-7111-5P, has-miR-509-3-5P, has-miR-5580-3P \\
ACTR3 & 7 & has-miR-1290, has-miR-5580-3P \\
STAT1 & 6 & has-miR-6825-5P,has-miR-4495, has-miR-5580-3P \\
MYH11 & 6 & has-miR-4495, has-miR-1290 \\
LAMC2 & 6 & miR-5580-3P \\
YWHAZ & 6 & has-miR-6825-5P, has-miR-1290, miR-5580-3P \\
YWHAQ & 6 & has-miR-6825-5P, has-miR-509-3-5P \\
HIF1A & 6 & has-miR-4495, has-miR-509-3-5P \\
\hline
\end{tabular}

Table 4. Functional (Biological Process) Enrichment Analysis of the Genes in the Module

\begin{tabular}{llcc}
\hline Category & Term & Count & $P$-value \\
\hline GOTERM_BP_FAT & GO:0044085 cellular component biogenesis & 7 & $9.70 \mathrm{E}-03$ \\
GOTERM_BP_FAT & GO:0002070 epithelial cell maturation & 2 & $1.50 \mathrm{E}-02$ \\
GOTERM_BP_FAT & GO:0051130 positive regulation of cellular component organization & $1.50 \mathrm{E}-02$ \\
GOTERM_BP_FAT & GO:0045926 negative regulation of growth & 5 & $1.50 \mathrm{E}-02$ \\
GOTERM_BP_FAT & GO:0010941 regulation of cell death & 3 & $2.60 \mathrm{E}-02$ \\
GOTERM_BP_FAT & GO:0006915 apoptotic process & 5 & $3.20 \mathrm{E}-02$ \\
\hline
\end{tabular}

targeted by three miRNAs (Table 5).

\section{Discussion}

Despite the increasing investigations on OSCC, the early diagnosis and treatment of it is still an issue due to the lack of understanding of the molecular and cellular mechanisms that drive the occurrence and progression of OSCC. Therefore, the need for in-depth studies into the molecular mechanisms of OSCC development is increasingly felt for OSCC prediction, early diagnosis and treatment. In current study, a total of 97 DEGs were identified. By constructing the PPI, we identified high degree genes including STAT1, ACLY, HIF1A, ENO1, CD44, YWHAQ, YWHAZ, LAMC2, ACTR3 and MYH11, which among them ACTR3 and MYH11 were down-regulated genes and the others were up-regulated ones.

STAT1 as another hub gene is the main component in interferon (IFN)-signaling that is involved in cellular functions in response to cytokines. Some clinical studies reported that STAT1 apply tumor promoter effects (Zhang and Liu, 2017). Moreover, there are also some reports claiming that STAT1 activation in squamous cell cancer of the oral cavity is a potential predictive marker of response to adjuvant chemotherapy (Laimer et al., 2007). In this study, ATP citrate lyase (ACLY) has also been introduced as another hub gene that is involved in lipid biogenesis linked with glucose metabolism. Studies have reported a tumorigenesis role for ACLY. In several cancer types (Migita et al., 2008; Qian et al., 2015; Teng et al., 2018), the ACLY has been expressed in higher levels in comparison to normal cells and its inhibition is known to induce proliferation arrest in cancer cells both in vitro and

Table 5. Significantly DEMs in OSCC Tissue and Their Potential Target Genes

\begin{tabular}{|c|c|c|}
\hline miRNAs & $\operatorname{logFC}$ & Target Genes \\
\hline has-miR-let-7d-3p & 0.79 & PARP11, HMGA2, SH3RF1, MEX3C, SEC24D, BEND2, PTGIS, PRKACB, GSTK1, PHF14 \\
\hline has-miR-6825-5p & 0.93 & NACC1, ENO1, BACH2, RIMS3, VAT1, STAT1, BCAS1, CDK18, YWHAZ, YEHAQ \\
\hline has-miR-7111-5p & 1.01 & PPP1R9B, CD44, WIZ, SLC6A17, NFIX, NR1D1, ACLY, ELK1, SRF, CASTOR2 \\
\hline has-miR-4495 & 1.15 & MYH11, PIAS2, HIF1A, TMEM33, EXTL2, STAT1, PIEZO2, AZIN1, GPR173, IKZF2 \\
\hline has-miR-1290 & 1.36 & SGO1, ACTR3, RTKN2, OSBPL6, ACER3, MYH11, AKAP2, YWHAZ-AKAP2, GTF2I \\
\hline has-miR-509-3-5P & -1.26 & CALD1, SERTAD2, AGFG1, ACLY, HIF1A, YWHAQ, DOCK3, CCSER2, CD200R1, FIGN \\
\hline has-miR-617 & -1.62 & RFXAP, CEP350, STXBP1, DGKH, CMC1, PDGFRL, EVI2A, PRPS2, AKT3, PPA1 \\
\hline has-miR-509-3P & -1.43 & ST3GAL2, OSBP, DDAH1, MMD2, PIP5K1B, VEZF1, RAB5C, RNF130, HABP4, RFX3 \\
\hline has-miR-6510-3p & -1.9 & HDAC3, SMURF2, NDFIP1, TSPAN17, AEN, ARID1B, C17orf107, CTIF, PCSK6, MRS2 \\
\hline has-miR-5580-3p & -1.92 & CD44, BTAF1, ACLY, DENR, ACTR3, STAT1, RASSF8, LAMC2, PDE10A, YWHAZ \\
\hline
\end{tabular}

$\overline{F C}$, fold change; Positive $\log \mathrm{FC}$ values denote up-regulated miRNAs, while negative $\log \mathrm{FC}$ values denote down-regulated miRNAs. If there were more than ten genes predicted by miRDB, only ten genes were listed in the Table 
a)
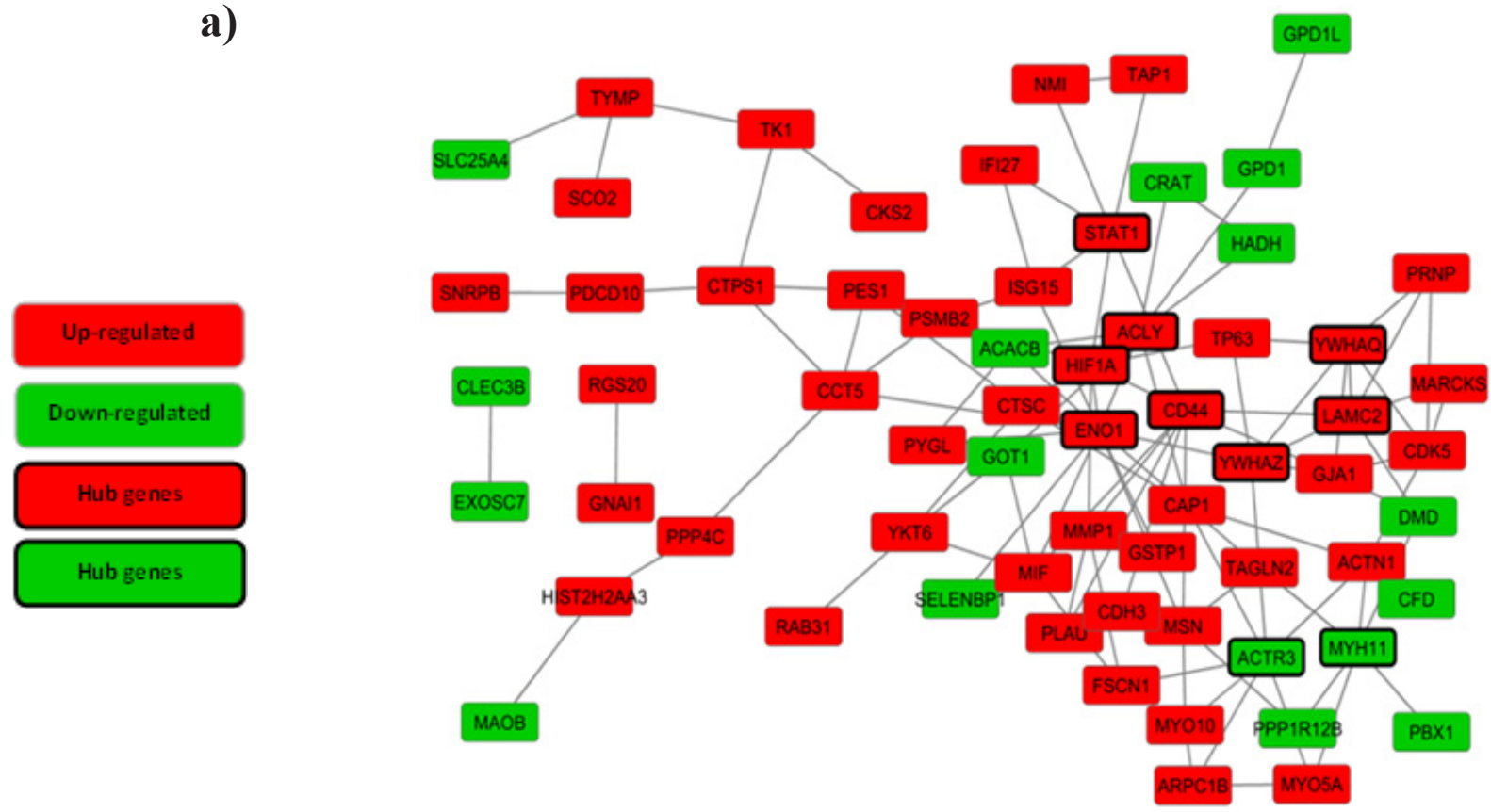

b)

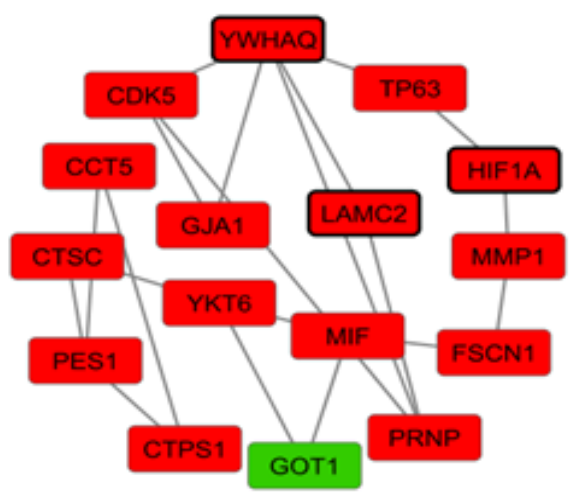

Figure 1. Protein-Protein Interaction (PPI) Network and Hub Genes. a) PPI network of differentially expressed genes (DEGs). b) A significant module selected from the PPI network. Red nodes denote up-regulated genes, while green nodes denote down-regulated genes. Black border shows that the gene is a hub. The lines represent an interaction relationship between the nodes.

in vivo (Zaidi et al., 2012). There are also evidences that present ACLY as a promising target for cancer treatment. Zhi et al., (2012) found that patients with tumors located in the oral cavity showed higher levels of ACLY gene in the tumor tissues compared to the healthy tissues (Zhi et al., 2015).

Another gene introduced as hub gene in this study was hypoxia-inducible factor-1 (HIF-1) that has been identified as a significant cancer drug target. Recent studies have found evidences of strong correlation between increased levels of HIF-1 and tumor metastasis, angiogenesis, and tumor resistance therapy (Masoud and Li, 2015). Recent researches in cancer biology at the cellular and molecular levels claimed that the HIF-1 $\alpha$ pathway is a main survival pathway for which novel strategies of cancer therapy could be developed. There is also a recent meta-analysis study demonstrated that HIF-1 $\alpha$ overexpression is associated with tumor size, tumor stage, lymph node metastasis, and overall survival of patients with OSCC. However, HIF-1 $\alpha$ could be used as an independent prognostic marker in patients with OSCC (Eckert et al., 2010; Zhou et al., 2017).
Another hub gene named ENO1 is also play an important role in tumorigenesis, cancer invasion, and metastasis (Capello et al., 2011; Gao et al., 2013; Zhao et al., 2015; Dai et al., 2017). regarding oral cancer, Ito et al., investigated differential expression of the human ENO1 gene in oral epithelium and OSCC, and results indicated that differential subcellular localization of ENO1 products may be closely related to carcinogenesis of the oral epithelium (Ito et al., 2007).

Another hug gene, $\mathrm{CD} 44$, is a non-kinase transmembrane glycoprotein that has various roles in cellular process such as cell division, migration, adhesion, and signaling. CD44 has been shown to be involved in tumor growth and metastasis and has also been reported as a cancer stem cell (CSC) marker in head and neck squamous cell cancer (HNSCC) (Chen et al., 2014). A study assessed immunohistochemical expression of CD44 in different grades of OSCC to evaluate its role in cancer progression showed an altered expression level of $C D 44$ in OSCC with weak immunostaining in poorly differentiated squamous cell carcinoma. Also, they suggested that the 
loss of cell adhesion which is correlated to the decrease of CD44 expression might be used in determination of OSCC progression (Kaza et al., 2018).

14-3-3 protein theta has also been introduced as hub that previous studies have reported that over-expression of $14-3-3 \zeta$ is an early event in oral tumorigenesis and may have a significant role in its development and progression. Thus, 14-3-3 $\zeta$ might serve as a potential therapeutic target for oral cancer (Matta et al., 2007).

Laminin $\gamma^{2}$ (LAMC2) is a subunit of the heterotrimeric glycoprotein laminin-332 that is a major component of epithelial basement membranes and play an important role in regulation of cell motility and adhesion (Marinkovich, 2007). It has been reported that LAMC2 was overexpressed in various carcinomas (Koshikawa et al., 1999; Yamamoto et al., 2001; Takahashi et al., 2002).

The last two hub genes with low expression in oral cancer were including ACTR3 and MYH11. ACTR3 is a member of the ARP2/3 complex. Previously, investigations in the field of metastasis has been focused on the actin cytoskeleton and high expression of actinrelated protein (ARP) during metastasis. Arp2/3 subunits have been reported by immunohistochemistry to be overexpressed in various cancer types, including lung (Semba et al., 2006), breast (Iwaya et al., 2007), gastric (Zheng et al., 2008) and colorectal cancers (Oser et al., 2009). Cortactin interacts with the Arp $2 / 3$ complex that there is a study showing that overexpression of cortactin in patients is related to high grade tumors, metastasis and poor survival in squamous cell carcinomas (Yamada et al., 2010; Sugahara et al., 2011).

The latest down-regulated hub gene was myosin-11 (MYH11), which is a smooth muscle myosin belonging to the myosin heavy chain family. Myosin MYH11 were previously found to be associated with variety of cancers, such as oral squamous cell carcinoma, meanwhile, a recent study has described $M Y H 11$ as a potential biomarker and candidate drug target for head and neck cancer management (Islam et al., 2018). Above all, these results support our findings that these hub genes are involved in the pathogenesis of carcinoma by affecting cell proliferation, cell migration, and metastasis.

Increasing evidence has indicated that the dysregulation of miRNAs play a significant role in the pathogenesis of variety of cancer types, including OSCC. In this study, we screened top 10 DEMs, including 5 up-regulated and 5 down-regulated miRNAs in OSCC. Has-miR-5580-3p is one of the most significantly down-regulated miRNAs and was found to target hub genes including CD44, ACLY, ACTR3, STAT1, YWHAZ and LAMC2. Additionally, has-miR-1290 is the main up-regulated miRNA that potentially targets $A C T R 3, M Y H 11$, and $Y W H A Z$. A recent report shows that has-miR-5580-3p is down-regulated in OSCC samples, esophagus squamous cell carcinoma tissues and colorectal cancer compared with healthy control (Wang et al., 2017; Chamorro-Petronacci et al., 2018b; Cheng et al., 2019). Has-miR-1290, which is one of the most frequently and consistently up-regulated miRNAs in human cancer, has been suggested as crucial drivers for tumor initiation and cancer progression (Zhang et al., 2016), and play an oncogenic role in cellular processes of esophageal squamous cell carcinoma (ESCC) (Li et al., 2015). Recently, Zhang et al., reported that direct inhibition of Has-miR-1290 with locked nucleic acid administered systemically, can arrest the growth of established patient-derived xenograft tumours, implying that this miRNA is clinically helpful as potential biomarker for follow up disease progression and as therapeutic target (Zhang et al., 2016). Furthermore, research observations indicated that, miR-1290 can behave as non-invasive biomarkers that could be used as early detection, prognostic and diagnostic biomarker for broad spectrum of cancers (Mo et al., 2015; Kobayashi et al., 2018). In case of oral cancer, Nakashima et al. found that miR-1290 expression level was significantly lower in the plasma of oral squamous cell carcinoma patients than in that of healthy, however, circulating miR-1290 status suggested as useful biomarker to predict the clinical response to chemoradiotherapy as well as overall survival in oral squamous cell carcinoma patients (Nakashima et al., 2019). As we found that most of hub genes (including CD44, ACLY, ACTR3, STAT1, LAMC2 and YWHAZ) were potentially targeted by has-miR-5580-3p, it indicates that this miRNA might play a key role in OSCC. In addition, according to Table 3, STAT1 was targeted by three miRNAs including has-miR-6825-5P, has-miR-4495 and has-miR5580-3P, indicating that these miRNAs play a significant role in OSCC development by mediating STAT1.

In conclusion, our study attempted to identify DEGs using comprehensive bioinformatics analyses and reported potential biomarkers for predicting the diseases progression. Our analysis has provided new points into OSCC pathogenesis by analyzing the hub genes and their interactions with miRNAs and consequently presenting the gene expression pattern, which can serve as candidate biomarkers and targets for potential treatment of OSCC. The limitation of this work is the lacking of verification experiments that could validate these predicted results obtained from bioinformatics analysis by using procedures such as qRT-PCR and Western Blot.

\section{Acknowledgements}

This project was supported by Proteomics Research Center of Shahid Beheshti University of Medical Sciences.

\section{Authors, contributions}

N. Amiri-Dashatan, N. Ahmadi, M. Rezaei-Tavirani and M. Koushki conceived the study and participated in its design and coordination. N. Amiri-Dashatan and N. Ahmadi carried out the experiment. N. Amiri-Dashatan drafted the original manuscript. All authors revised the manuscript and approved the final manuscript.

\section{References}

Atan NAD, Koushki M, Tavirani MR, et al (2018). Protein-protein interaction network analysis of salivary proteomic data in oral cancer cases. Asian Pac J Cancer Prev, 19, 1639-45.

Atan NAD, Yekta RF, Nejad MR, et al (2014). Pathway and network analysis in primary open angle glaucoma. JPS, 5, 92-101. 
Basakran NS (2015). CD44 as a potential diagnostic tumor marker. Saudi Med J, 36, 273-9.

Capello M, Ferri-Borgogno S, Cappello P, et al (2011). $\alpha$-enolase: a promising therapeutic and diagnostic tumor target. FEBS J, 278, 1064-74.

Chakraborty S, Nagashri M, Mohiyuddin SA, et al (2010). Gene expression profiling of oral squamous cell carcinoma by differential display rt-PCR and identification of tumor biomarkers. Indian J Surg Oncol, 1, 284-93.

Chamorro-Petronacci C, Perez-Sayáns M, Padín-Iruegas ME, et al (2018a). Differential expression of snoRNAs in oral squamous cell carcinomas: new potential diagnostic markers. $J$ Enzyme Inhib Med Chem, 33, 424-7.

Chen C, Méndez E, Houck J, et al (2008). Gene expression profiling identifies genes predictive of oral squamous cell carcinoma. Cancer Epidem Biomar, 17, 2152-62.

Cheng L, Shi G, Fang C, et al (2019). Identifying the differentially expressed microRNAs in esophagus squamous cell carcinoma of Kazakh patients in Xinjiang. Oncol Lett, 17, 2657-68.

Dai L, Qu Y, Li J, et al (2017). Serological proteome analysis approach-based identification of ENO1 as a tumor-associated antigen and its autoantibody could enhance the sensitivity of CEA and CYFRA 21-1 in the detection of non-small cell lung cancer. Oncotarget, 8, 36664-73.

Dashatan NA, Tavirani MR, Zali H, et al (2018). Prediction of Leishmania major key proteins via topological analysis of protein-protein interaction network. Galen Med J, 7, 1129.

Eckert AW, Schütze A, Lautner MH, et al (2010). HIF-1 $\alpha$ is a prognostic marker in oral squamous cell carcinomas. Int $J$ Biol Markers, 25, 87-92.

Estilo CL, Pornchai O, Talbot S, et al (2009). Oral tongue cancer gene expression profiling: Identification of novel potential prognosticators by oligonucleotide microarray analysis. BMC Cancer, 9, 11.

Gao J, Zhao R, Xue Y, et al (2013). Role of enolase-1 in response to hypoxia in breast cancer: exploring the mechanisms of action. Oncol Rep, 29, 1322-32.

Ge L, Liu S, Xie L, et al (2015). Differential mRNA expression profiling of oral squamous cell carcinoma by high-throughput RNA sequencing. J Biomed Res, 29, 397-404.

Islam T, Rahman R, Gov E, et al (2018). Drug targeting and biomarkers in head and neck cancers: insights from systems biology analyses. OMICS, 22, 422-36.

Ito S, Honma T, Ishida K, et al (2007). Differential expression of the human $\alpha$-enolase gene in oral epithelium and squamous cell carcinoma. Cancer Sci, 98, 499-505.

Iwaya K, Norio K, Mukai K (2007). Coexpression of Arp2 and WAVE2 predicts poor outcome in invasive breast carcinoma. Modern Pathol, 20, 339-43.

Kaza S, Kantheti LP, Poosarla C, et al (2018). A study on the expression of CD44 adhesion molecule in oral squamous cell carcinoma and its correlation with tumor histological grading. J Orofac Sci, 10, 42-9.

Koshikawa N, Moriyama K, Takamura H, et al (1999). Overexpression of laminin $\gamma 2$ chain monomer in invading gastric carcinoma cells. Cancer Res, 59, 5596-601.

Laimer K, Spizzo G, Obrist P, et al (2007). STAT1 activation in squamous cell cancer of the oral cavity: a potential predictive marker of response to adjuvant chemotherapy. Cancer, 110, 326-33.

Li M, He X-Y, Zhang Z-M, et al (2015). MicroRNA-1290 promotes esophageal squamous cell carcinoma cell proliferation and metastasis. World J Gastroenterol, 21, 3245-55.

Malik UU, Zarina S, Pennington SR (2016). Oral squamous cell carcinoma: Key clinical questions, biomarker discovery, and the role of proteomics. Arch Oral Biol, 63, 53-65.

Manikandan M, Rao AKDM, Arunkumar G, et al (2016). Oral squamous cell carcinoma: microRNA expression profiling and integrative analyses for elucidation of tumourigenesis mechanism. Mol Cancer, 15, 11.

Marinkovich MP (2007). Laminin 332 in squamous-cell carcinoma. Nat Rev Cancer, 7, 370-80.

Masoud GN, Li W (2015). HIF-1 $\alpha$ pathway: role, regulation and intervention for cancer therapy. Acta Pharm Sin B, 5, 378-89.

Matta A, Bahadur S, Duggal R, et al (2007). Over-expression of 14-3-3zeta is an early event in oral cancer. BMC Cancer, $7,11$.

Migita T, Narita T, Nomura K, et al (2008). ATP citrate lyase: Activation and therapeutic implications in non-small cell lung cancer. Cancer Res, 68, 8547-54.

Mo D, Gu B, Gong X, et al (2015). miR-1290 is a potential prognostic biomarker in non-small cell lung cancer. $J$ Thorac Dis, 7, 1570-79.

Momen-Heravi F, Bala S (2018). miRNA regulation of innate immunity. J Leukoc Biol, 103, 1205-17.

Nakashima H, Yoshida R, Hirosue A, et al (2019). Circulating miRNA-1290 as a potential biomarker for response to chemoradiotherapy and prognosis of patients with advanced oral squamous cell carcinoma: A single-center retrospective study. Tumor Biol, 41, 10.

Oser M, Yamaguchi H, Mader CC, et al (2009). Cortactin regulates cofilin and N-WASp activities to control the stages of invadopodium assembly and maturation. $J$ Cell Biol, 186, 571-87.

Park EJ, Shimaoka M, Kiyono H (2017). MicroRNA-mediated dynamic control of mucosal immunity. Int Immuno, 29, 157-63.

Qian X, Hu J, Zhao J, et al (2015). ATP citrate lyase expression is associated with advanced stage and prognosis in gastric adenocarcinoma. Int J Clin Exp Med, 8, 7855-60.

Schneider A, Victoria B, Lopez YN, et al (2018). Tissue and serum microRNA profile of oral squamous cell carcinoma patients. Sci Rep, $\mathbf{8}, 8$.

Semba S, Iwaya K, Matsubayashi J, et al (2006). Coexpression of actin-related protein 2 and Wiskott-Aldrich syndrome family verproline-homologous protein 2 in adenocarcinoma of the lung. Clin Cancer Res, 12, 2449-54.

Sugahara K, Michikawa Y, Ishikawa K, et al (2011). Combination effects of distinct cores in 11q13 amplification region on cervical lymph node metastasis of oral squamous cell carcinoma. Int J Oncol, 39, 761-9.

Takahashi S, Hasebe T, Oda T, et al (2002). Cytoplasmic expression of laminin $\gamma 2$ chain correlates with postoperative hepatic metastasis and poor prognosis in patients with pancreatic ductal adenocarcinoma. Cancer, 94, 1894-901.

Teng L, Chen Y, Cao Y, et al (2018). Overexpression of ATP citrate lyase in renal cell carcinoma tissues and its effect on the human renal carcinoma cells in vitro. Oncol Lett, 15, 6967-74.

Wang Y-n, Chen Z-h, Chen W-c (2017). Novel circulating microRNAs expression profile in colon cancer: a pilot study. Eur J Med Res, 22, 11.

Yamada S-i, Yanamoto S, Kawasaki G, et al (2010). Overexpression of cortactin increases invasion potential in oral squamous cell carcinoma. Pathol Oncol Res, 16, 523-31.

Yamamoto H, Itoh F, Iku S, et al (2001). Expression of the $\gamma 2$ chain of laminin-5 at the invasive front is associated with recurrence and poor prognosis in human esophageal squamous cell carcinoma. Clin Cancer Res, 7, 896-900.

Zaidi N, Swinnen JV, Smans K (2012). ATP-citrate lyase: a key player in cancer metabolism. Cancer Res, 72, 3709-14.

Zhang WC, Chin TM, Yang H, et al (2016). Tumour-initiating 
cell-specific miR-1246 and miR-1290 expression converge to promote non-small cell lung cancer progression. Nat Commun, 7, 16.

Zhang Y, Liu Z (2017). STAT1 in cancer: friend or foe?. Discov Med, 24, 19-29.

Zhao M, Fang W, Wang Y, et al (2015). Enolase-1 is a therapeutic target in endometrial carcinoma. Oncotarget, 6, 15610-27.

Zheng H-C, Zheng Y-S, Li X-H, et al (2008). Arp2/3 overexpression contributed to pathogenesis, growth and invasion of gastric carcinoma. Anticancer Res, 28, 2225-32.

Zhi X, Lamperska K, Golusinski P, et al (2015). Gene expression analysis of head and neck squamous cell carcinoma survival and recurrence. Oncotarget, 6, 547-55.

Zhou J, Huang S, Wang L, et al (2017). Clinical and prognostic significance of HIF- $1 \alpha$ overexpression in oral squamous cell carcinoma: a meta-analysis. World J Surg Oncol, 15, 8.

\section{c) (7) (2)}

This work is licensed under a Creative Commons AttributionNon Commercial 4.0 International License. 